\title{
XLVI. A new account of the genus Echeveria
}

\section{A.H. Haworth Esq. F.L.S.}

To cite this article: A.H. Haworth Esq. F.L.S. (1828) XLVI. A new account of the genus Echeveria, Philosophical Magazine Series 2, 4:22, 261-264, DOI: 10.1080/14786442808674807

To link to this article: http://dx.doi.org/10.1080/14786442808674807

曲 Published online: 10 Jul 2009.

Submit your article to this journal $\pi$

Џll Article views: 3

Q View related articles $₫$ 


\section{$\left[\begin{array}{ll}261 & ]\end{array}\right.$}

XLVI. A new Account of the Genus Echeveria. By A. H. Haworth, Esq. F.L.S. \&c. \&c.

To the Editors of the Philosophical Magazine and Annals.

Gentlemen,

$\mathbf{H}^{\mathrm{A}}$

AVING just examined and described a fine new succulent

plant at Mr. Tate's Nursery in Sloane-street, which he has recently raised from Mexican seeds, and which is now blooming for the first time in Europe, amongst many other equally rare and well-managed plants; I send you hereunder a full account of it, and four other species of the same genus; to which I have added all their synonyms.

This new plant, belonging to DeCandolle's new genus Echeveria, I have called, from its ample leaves, Echeveria grandifolia.

In my ninth decade of new succulent plants, published in your Journal in 1826, I announced to you (inter alia) that Cotyledon coccinea of Cavanilles, and Cotyledon umbilicus of Linné, would each form the type of a new genus, but for want of proper specimens regretted my inability at that time to give you sufficient characters. This, however, is now the less to be lamented, as DeCandolle has done it for us, in the third volume of his Prodromus, just published; and to the above-mentioned Cotyledon coccinea has added my Cotyledon caspitosa, (which is a native of California, although we used to think it African,) and two new species from Mexico.

If you can find room for this communication in the next Number of your Journal, you will much oblige

Your old correspondent and friend,

Chelsea, Aug. 10, 1828.

A. H. Hawonth.

\section{Ordo Naturalis.}

Crassulacea DeCandolle. Sempervive Juss. \&c.-Cotyledones americance Auctorum.

Genus, Echeveria DeCand. Prod. 3.' 401.

Generis Character.

Calyx 5-partitus, sepalis distantibus valdè foliiformibus, præinæqualibus basi coalitis. Corolla pentapetaloidea pentagona campanularis. Petala infernè concreta erecta rigidula acuta crassa, basi inter calycis folia, extùs gibba, intùs scrobiculata: duobus exterioribus petalis insuper tria interiora, arctè imbricatim adpressa. Stamina 10, basi cum petalis longioribus concreta. Squame ordinaria 
breves subquadratæ albæ, alternæ crassiores cerinæ, omnes in petalorum scrobiculis nidulantes. Carpella 5 erecta, in stylos acutos desinentia.

Suffrutices Mexicani parvi succulenti glauci. Folia basi soluta: rosularia sed alterna, integra, cum mucronulo, at sæpiùs valdè obtusa, et in spicis florigeris foliolosis, pedetentim in bracteas numerosas magnas subdistantibus omninò foliiformes abeuntia. Flores valdè bracteati, spicati, s. paniculati, vel cymosi, et tunc secus cymæ ramos sessiles, coccinei flavive.

\section{Specienum Characteres.}

* Suffrutices, floribus paniculatis spicatisve, coccineis.

grandifolia. E. (great-leaved) foliis orbiculato-cuneatis grossè

1. petiolatis, floribus paniculato-spicatis.

Habitat in Mexico.

Floret Aug. Sept. G. H. Ћ.

Caudex in nostro exemplo, in caldario, apud Dom. Tate, in secundo anno triuncialis diametro subunciali, cylindricus carnoso-lignescens radiculos exiguos terram versus exerens. Folia numerosa conferta ambienter multifaria, seu in rosulam laxam digesta, patenti-recurvula dodrantalia plùsve incurvo-concavula, et in petiolum carnosum subsemunciam crassum obtusè canaliculatum attenuata, pruinoso glauca rufo marginata integra rariùsve minutim asperiuscula; subtus, basin versus præcipuè vivacitèr glauco-purpurascentia: et denique morientia inania lorea persistentia. Florum paniculæ sesquipedales, bracteatim foliolosæ, axillares teretes uti folia cæruleo-glaucæ; bracteis erectis lanceolatis mucronulatis (magì quàm vera folia) distantibus sensim sensimque minoribus, et Sedi more singulariter basi planè obtusèque solutis. Calyx sepalis 5 valdè inæqualibus bracteis brevioribus omninò foliiformibus (excepto basi non soluto) tribus cæteris duplò majoribus, quarto minore, quinto minuto. Corolla ferè semunciam longa, calyce brevior rubro-aurantiaca, rore roseo-glauca purpureave. Stamina 10, petalis humiliora alba, antheris erectis polline luteo. Carpella grossa, alba in stylos virides abeuntia. Cætera ferè ut in $E$. coccineâ, infrà descripta, at non rectè vidi.

gibbiflora. E. (gibbous-flowered) foliis planis cuneiformibus

2. acutè mucronatis ad apices ramorum confertis, paniculâ patente, floribus secus ramos breviter pedicellatis. DeCand. Prod. 3. p. 401. 
Habitat in Mexico. $h$.

Petala basi albida, apice subcoccinea. DeCand.l.c.

coccinea. E. (pubescent) mollis : ramulis foliisque spatulato-

3. lanceolatis pallescente densè puberulis, florum spicis axillaribus elongatis foliolosis.

Cotyledon coccineum. Cav. Ic. 2. t. 170.-Nob. in Suppl. Pl. Succ. p. 26. A.D. 1819.-Lodd. Bot. Cab. t.832.-Echeveria coccinea. DeCand.Prod.v.3.p.401.

Habitat in Mexico.

Floret autumno, hyemeve. G. H. $\hbar$.

Suffrutex sesquipedalis, parùm et alternè ramosus. Flores densè elongato-spicati, spica sub foliorum capitulos adscendentes thyrsiformes bracteatim foliolosæ, supernè ferè comosæ, post florescentiam longum per tempus induratè denudatæ persistentes. Flores duplò minores quàm in $\boldsymbol{E}$. grandifoliâ, horizontaliter sessiles. $\boldsymbol{C} a$ lycis sepala subovato-lanceolata acuminata tumidè carnosa patenti-recurvula et inter bracteas irregulariter intertexta. Corolla 5-petaloidea campanulata coccinea, infernè pentagona, basi gibbulis 5 , e foveolis totidem internis nectariferis : laciniæ (corollæ) rectæ ovato-lanceolatæ,acuminatæ calyce breviores carinâ densiùs ciliatopuberulâ saturatiore; intùs longè pallidiores glabræ foveolatæ. Stamina 10, albo-Iutescentia corollẩ subdimidiatim breviora, 5 exteriora germinum basi inserta, 5 alia in foveolâ supradictâ dimidiatim flexuosè adnata, solùm supernè libera, et 5 prioribus (staminibus) aliquantillum humiliora. Antherce erectæ subparallelipipedæ emarginatæ, basi cordatæ, polline luteo. Carpella 5 , erecto-adpressa, cum stylis continuantibus obclavatis luteis, stigmate purpureo inconspicuo, lente hemisphærico, et subinde ad lucem pellucente.

Obs. Corolle laciniæ intùs argutè canaliculatæ, in quibus canaliculis insident exteriora filamenta, et ad eas adpressa, atque ad earum flexionem gibber parvus exstat utraque insuper apicem singulæ foveolæ supradictæ.

Ad basin singuli carpelli rudimentum solum squamulæ ordinariæ exstat tumidulum rhombeum subquadratumve, carpello omninò adnatum, pustulam minutissimam simulante. Seminula numerosa incipientia oblonga alba solùm vidi.

teretifolia. E. (cylindric-leaved) foliis teretibus acutis sparsis 4. basi subsolutis, spicis secundis paucifloris. DeCand. Prod. 3. p. 401. 
264 Mr. Haworth's Description of the Genus Echeveria.

Habitat in Mexico. そ. Flos omninò prioris. De Cand. 1. c.

** Subherbaceæ, floribus subcymosis lnteis.

ccespitosa. E. (dwarf yellow-flowered) foliis rosularibus an-

5. gustè linguiformibus, apice obcuneatis submucronatis, floribus cymosis.

Cotyledon cæspitosa. Nob. Misc. Nat. p. 180: A.D. 1803.-Cotyledon linguiformis. Ait. Hort. Kew. v. 3. p. 109.-Sedum Cotyledon. Jacq. f. Eclog. 1. f. 17.-Cotyledon reflexum. Willd. Enum. Suppl. p. 24.-Echeveria cæspitosa. DeCand. Prod. v. 3. p. 401 .

Habitat in Californiâ.

Floret Jul. Aug. G. H. భ. s. 々.

P.S. I avail myself of the present opportunity of correcting the following errors, which time alone has enabled me to ascertain.

1. Mesembr. deflexum, $\beta$. Revis. Pl. Succ. p. 140, is the same as $M$. imbricans, p. 139, and the last is a good and most abundant-flowering species.

2. M. leptaleon is the young state only of M: retroflexum, l.c.

3. $M$. flexile is the young state only of $M$. polyphyllum, l. c.

4. And $M$. torquatum is a casual state only of M. floribundum. Revis. Pl. Succ. p. 187.

DeCandolle in vol. 3. p. 416 of his Prodromus, just published, says of Mesembryanthema: "Species pleræque hortenses ex cl. Haworth, \&c.- - sed forsan plures ut meræ varietates habendæ."

But all the new species which I have published, I was well and sufficiently assured, were raised from wild African, or Australasian seeds, except the following only, whose origin I cannot trace further than I have printed. M. ficiforme; M. hybridum; $M$. nobile; $M$. mustellinum; M. bigibberatum; M. cruciatum; M. Salmii; M. cultratum; M. coruscans; $M$. procumbens; $M$. variabile; M. mucroniferum; M. loratum, and $M$. hispifolium. And even seven of the above I received from His Highness the Prince de Salm Dyck, whose genuine origin I believe he can point out.

In my last communication Phil. Mag. and Annals, N.S., vol. iii. p. 184, l. 15, for Crassulam undosam, read Crassulam undatam. 